\title{
Miíase Oral: etiologia, diagnóstico e tratamento
}

Oral Myiasis: etiology, diagnosis and treatment

\section{Alessandro Leite Cavalcanti}

\section{Resumo}

A miíase oral é uma doença causada pela infestação dos tecidos por larvas de moscas. As manifestações clínicas da doença não são específicas e variam de acordo com a região do corpo envolvida. $\mathrm{O}$ tratamento consiste na remoção mecânica das larvas, sendo recomendado o uso sistêmico de ivermectina. Este artigo traz uma revisão da literatura a respeito desta doença, enfatizando aspectos relativos à etiologia, diagnóstico e tratamento da miíase oral.

Palavras-chave: Miíase, Ectoparasitoses, Doenças parasitárias

\begin{abstract}
Oral myiasis is a disease caused by the invasion of tissues by larvae of flies. Clinical manifestations of myiasis are not specific and vary according to the involved area of the body. The treatment is the mechanical removal of the maggots and recommendation of the use of systemic ivermectin. This paper makes a review of the literature, emphasizing etiological aspects, diagnosis and treatment of oral myiasis.
\end{abstract}

Keywords: Myiasis, Ectoparasitic infestations, Parasitic diseases
${ }^{1}$ Professor Doutor do Departamento de Odontologia da Universidade Estadual da Paraíba (UEPB).

Correspondência: Alessandro Leite Cavalcanti

Endereço: Avenida Manoel Moraes, 471/802 - Manaíra CEP: 58038-230

João Pessoa, PB

E-mail: dralessandro@ibest.com.br

\section{Introdução}

As transformações introduzidas pelo homem nos ambientes primitivos e a criação extensiva de animais domésticos (bovinos, equídeos, ovinos, suínos e aves) têm propiciado a adaptação e a proliferação de moscas nos ambientes antrópicos (CHICARELLI et al., 2002). Alguns tipos de moscas podem ocasionar doenças em animais e seres humanos. Entre os animais, as moscas identificadas como varejeiras - C. hominivorax - acarretam o mal conhecido como bicheira entre os pecuaristas.

Em humanos, conforme a área afetada no local do corpo acometido, a miíase pode ser subdividida em: cutânea, de órgãos internos (intestino e sistema urinário) e de cavidades (ocular, nasal, oral, vaginal e anal) (GUTIERREZ, 1990; HALL; WALL, 1995; VICTORIA; TRUJILLO; BARRETO, 1999). A miíase oral é uma condição que pode se manifestar em bolsas periodontais, feridas resultantes de exodontias, feridas abertas em geral, em diversas localizações dentro da cavidade oral (LEAL et al., 2001; GURSEL et al., 2002; SHARMA; MAMATHA; ACHARYA, 2008).

Este tipo de infecção ocorre principalmente em áreas tropicais (CENCIL et al., 2006) e está associada a uma higiene corpórea e oral inadequadas (GURSEL et al., 2002). Moscas adultas são fortemente atraídas por odores emitidos por tecidos putrefatos, depositando ovos em áreas necróticas de feridas, os quais originam larvas que se alimentam do hospedeiro vivo ou morto (KHAYAT, 2002).

Embora médicos (otorrinolaringologistas e dermatologistas) e veterinários tenham maior vivência com esta patologia (FREITAS et al., 1989), o cirurgião-dentista deve estar atento à sua presença, posto que existem vários relatos de casos de miíase na cavidade oral envolvendo crianças e adultos na literatura brasileira (ABDO et al., 2006; CHICARELLI et al., 2002; FIGUEIREDO et al., 2000; LEAL etal., 2001;PONTES etal., 2002; ROSSI-SCHNEIDER et al., 2007; OLIVEIRA; MACHADO; OLIVEIRA, 2008) e estrangeira (BHATT; JAYAKRISHNAN, 2000; JOO; KIM, 2001; GURSEL et al., 2002; SCOTT, 1964; KHAYAT, 2002; YAZAR et al., 2005; DROMA et al., 2007; SHARMA et al., 2008).

Portanto, com base no exposto acima e face à ocorrência desta patologia na cavidade oral, este trabalho tem por objetivo apresentar, por meio de uma revisão de literatura, aspectos relativos à prevalência, etiologia, às características clínicas e ao tratamento da miíase oral. 


\section{Metodologia}

A revisão da literatura internacional foi realizada na Base de Dados PubMed utilizando-se os descritores myiasis e oral, no período de 1963 a 2009. Para avaliação da literatura nacional, as Bases de Dados BBO (Bibliografia Brasileira de Odontologia) e LILACS (Literatura Latino-Americana e do Caribe em Ciências da Saúde) foram utilizadas, sendo empregado o descritor miíase e o período de busca compreendeu os anos de 1985 a 2009.

Foram encontrados 119 artigos internacionais e 130 nacionais, totalizando 249 trabalhos. Após a leitura do título e resumo dos artigos, 35 trabalhos foram selecionados. Como critérios de inclusão, foram selecionados relatos de casos acometendo a cavidade oral e as revisões de literatura que abordavam o tema. Os artigos que não se encaixavam nos critérios acima foram excluídos. Incluiu-se ainda a consulta a um livro sobre doenças parasitárias.

\section{Revisão de literatura}

\section{Etimologia}

O termo miíase tem origem na palavra grega myia e significa mosca, sendo utilizado para designar a invasão de tecidos vivos em seres humanos e outros mamíferos (KHAYAT, 2002; MILIKAN, 1999; GURSEL et al., 2002) por larvas de dípteros (CHICARELLI et al., 2002; MELO et al., 2000; YAZAR et al., 2005). O primeiro relato existente na literatura foi feito por Laurence, em meados do século $X X$, mais precisamente no ano de 1909 (BHATT; JAYAKRISHNAN, 2000).

As moscas responsáveis por esta condição preferem um ambiente quente e úmido, de modo que a miíase está restrita aos meses de verão em zonas de clima temperado, porém pode ocorrer em todos os meses do ano em países tropicais e subtropicais (FERRO et al., 2003; MEINKING; BURKHART; BURKHART, 2003; DROMA et al., 2007).

\section{Prevalência e Etiologia}

Nos Estados Unidos, de acordo com Scott (1964), a prevalência de miíase na cavidade oral é extremamente baixa, visto que, ao estudar 120 casos de miíase em humanos, apenas um único paciente $(0,9 \%)$ foi acometido na cavidade oral. No Brasil, estudo epidemiológico desenvolvido em um hospital do município de Uberlândia, MG, revelou que de um total de 431 prontuários médicos examinados em um período de dez anos (1986-1997), 222 (51,5\%) casos de miíase foram diagnosticados, dos quais apenas $2(0,9 \%)$ acometeram a cavidade oral (FERRO et al., 2003). Em relação à distribuição entre os sexos, esta patologia é mais comum no sexo masculino e apesar de acometer todas as faixas etárias (FERRO et al., 2003), a sua ocorrência em crianças pode ocasionar danos mais severos ou ser, até mesmo, fatal (LIM, 1974).

Mais de 150 espécies de dípteros podem causar miíase em humanos (CENCIL et al., 2006). Inúmeras são as espécies de moscas que podem infestar a cavidade oral, dependendo da distribuição geográfica dos dípteros (DROMA et al., 2007). No Brasil, por exemplo, foram descritas as espécies Sarcophagi (BOZZO et al., 1992) e Cochiliomia hominivorax (SHINOHARA et al., 2004; OLIVEIRA; MACHADO; OLIVEIRA, 2008; GEALH et al., 2009). Em Israel e na Turquia, as espécies Wohlfahrtia magnifica e Sarcophagidae (DROMA et al., 2007; AYDENIZÖZ; DIK, 2008) e em Hong Kong, Chrysomya bezziana (NG et al., 2003).

Em relação à etiologia, várias são as causas descritas na literatura para a infestação dos tecidos vivos por larvas de moscas e elas são geograficamente dependentes. Entretanto, fatores como higiene pessoal deficiente (KHAYAT, 2002; SHARMA et al., 2008), respiração bucal noturna (GURSEL et al., 2002; HAKIMI; YAZID, 2002; BARBOSA et al., 2008), resistência corporal diminuída, alcoolismo e doença periodontal avançada (DROMA et al., 2007; SHARMA; MAMATHA; ACHARYA, 2008), senilidade (ROSSI-SCHNEIDER et al., 2007), déficit neuropsicomotor (RAWLINS; BARNETT, 1983; KHAYAT, 2002), hipersalivação (RAWLINS; BARNETT, 1983), desnutrição, traumas e infecção na área bucomaxilofacial podem ser predisponentes ao aparecimento da doença (FREITAS et al., 1989; FIGUEIREDO et al., 2000; STEPHAN; FUENTEFRIA, 1999; SHARMA; MAMATHA; ACHARYA, 2008). São citados ainda o diabetes e a existência de doenças vasculares periféricas em pacientes idosos como possíveis fatores predisponentes da doença (JOO; KIM, 2001).

\section{Classificação}

No que concerne à invasão tecidual, a miíase pode ser classificada em três diferentes tipos (CHICARELLI et al., 2002; FIGUEIREDO et al., 2000; PONTES et al., 2002; KHAYAT, 2002):

Obrigatórias (biontófagas ou primárias): as larvas de dípteros se desenvolvem naturalmente sobre ou dentro de vertebrados, alimentando-se de tecidos vivos. Dentre suas espécies, encontram-se: Cochliomy homini vorax e Dermatobia hominis;

Facultativas (necrobiontófogas secundárias): aquela na qual as larvas de dípteros se desenvolvem em matéria orgânica em decomposição (cadáveres), atuando como saprófagas, mas podem, no entanto, acometer os tecidos necrosados em um hospedeiro vivo. Os gêneros mais comuns são: Phaenicia, Musca, Lucília e Fannia;

Pseudomíase (acidental): ocorre por ingestão de ovo e/ ou larvas de dípteros presentes em alimentos contaminados. Pode ocasionar distúrbios intestinais.

\section{Manifestações Clínicas e Diagnóstico}

As manifestações clínicas variam de acordo com a espécie e a área do corpo envolvida (HAKIMI; YAZID, 2002; CECIL et al., 2006), podendo configurar desde quadros benignos leves e assintomáticos (BHATT; JAYAKRISHNAN, 2000), até formas graves com sérias complicações, até mesmo a morte (MELO et al., 2000). Dentre os sinais e sintomas clínicos mais comumente descritos na literatura estão a presença de mialgia (KHAYAT, 2002; DROMA et al., 2007; CAVALCANTI et al., 2008), febre (ABDO et al., 2006; HAKIMI; YAZID, 2002), odor acentuado (ROSSI-SCHNEIDER et al., 2007) e desconforto local (STEPHAN; FUENTEFRIA, 1999). Além de inflamação dos tecidos circundantes, podem ser vistas ulcerações, necrose tecidual (CAVALCANTI et al., 2008) e até envolvimento ósseo (KONSTANTINIDIS; ZAMANIS, 1987). Os exames laboratoriais incluem a presença de hipereosinofilia e elevada taxa de sedimentação de eritrócitos (CENCIL et al., 2006).

A movimentação das larvas facilita o diagnóstico da miíase oral. Entretanto, na ausência dessa movimentação, por vezes, além do exame visual clínico, a exploração cirúrgica e o exame histopatológico levam ao diagnóstico final (STEPHAN; FUENTEFRIA, 1999). Caso o diagnóstico seja tardio e o número de larvas presentes na lesão demasiadamente elevado, pode ocorrer maior dano tecidual, requerendo, inclusive, a realização de cirurgia estética (ABDO et al., 2006).

\section{Localização}

As lesões geralmente estão localizadas no segmento anterior de ambos os maxilares (HAKIMI; YAZID, 2002; KHAYAT, 2002; SHINOHARA et al., 2004; SHARMA et al., 2008; CAVALCANTI et al., 2008) e no palato (NG et al., 2003; ROSSI-SCHNEIDER et al., 2007). No entanto, a região posterior também pode ser acometida (DROMA et al., 2007), bem como a língua (OLIVEIRA; MACHADO; OLIVEIRA, 2008). 


\section{Tratamento}

A conduta padrão a ser adotada para os casos de miíase oral é a remoção mecânica das larvas (ABDO et al., 2006; BHATT; JAYAKRISHNAN, 2000; HAKIMI; YAZID, 2002; OLIVEIRA; MACHADO; OLIVEIRA et al., 2008; SHARMA et al., 2008; BARBOSA et al., 2008), com o auxílio da cureta periodontal e pinça clínica, geralmente, sob anestesia ou analgesia, dependendo do estado clínico do paciente (DROMA et al., 2007; CAVALCANTI et al., 2008). A remoção das larvas deve ser realizada de modo cuidadoso, para que não sejam fragmentadas, e os seus restos permaneçam no local, servindo de meio para infecção (MELO et al., 2000). Esta remoção é um processo doloroso, incômodo e constrangedor, quer para o paciente quer para o profissional (CENCIL et al., 2006).

Contudo, quando existem múltiplas larvas e avançada destruição tecidual, a aplicação de substâncias tópicas asfixantes tem sido recomendada a fim de assegurar a completa remoção de todas as larvas (ABDO et al., 2006; ROSSI-SCHNEIDER et al., 2007), assim como o debridamento de tecidos necróticos (ABDO et al., 2006) e o seu restabelecimento cirúrgico (STEPHAN; FUENTEFRIA, 1999). Dentre as diversas substâncias reportadas na literatura, estão o uso do gel de digluconato de clorexidina a 0,2\% (FIGUEIREDO et al., 2000), solução de éter (LEAL et al., 2001; BHATT; JAYAKRISHNAN, 2000; CAVALCANTI et al., 2008), óleo de turpetine (SHARMA et al., 2008) e até mesmo a utilização de vaselina sólida (PONTES et al., 2002). Outras soluções, como iodofórmio e solução creosotada a 10\% como curativo tampão (FREITAS et al., 1989), também foram empregadas.

A literatura reporta ainda, com bastante sucesso, a utilização sistêmica de uma dose de ivermectina, um antibiótico macrolídeo semissintético para o tratamento de casos mais severos envolvendo a existência de miíase oral (ABDO et al., 2006; CENCIL et al., 2006; VICTORIA et al., 1999; SHINOHARA et al., 2004; OLIVEIRA; MACHADO; OLIVEIRA, 2008; SHARMA et al., 2008; GEALH et al., 2009). Em pacientes com deficiências nutricionais, recomenda-se o uso de complexos vitamínicos associados à administração de antibióticos (MELO et al., 2000).

\section{Discussão}

As péssimas condições locais de higiene, com ferimentos ou presença de secreções, são fatores favoráveis para que as moscas ou outros insetos que atuem como vetores mecânicos, depositem seus ovos e as moscas se desenvolvam (MELO et al., 2000; FERRO et al., 2003). Por conseguinte, as medidas de proteção, individuais e coletivas, são as principais formas para se prevenir a instalação da miíase (ABDO et al., 2006; FERRO et al., 2003; PONTES et al., 2002; OLIVEIRA; MACHADO; OLIVEIRA, 2008).

Inúmeros fatores são apontados na literatura como os principais agentes etiológicos da miíase oral, dentre os quais estão a higiene pessoal deficiente (KHAYAT, 2002; SHARMA et al., 2008; BARBOSA et al., 2008; CAVALCANTI et al., 2008), doença periodontal avançada (DROMA et al., 2007; SHARMA et al., 2008) traumas na região bucomaxilofacial (FREITAS et al., 1989; STEPHAN; FUENTEFRIA, 1999). A presença de senilidade (ROSSI-SCHNEIDER et al., 2007), déficit neuropsicomotor (RAWLINS; BARNETT, 1983; KHAYAT, 2002; SHINOHARA et al., 2004), diabetes e doenças vasculares periféricas (JOO; KIM, 2001) em pacientes idosos constituem-se também em possíveis agentes predisponentes da doença.

A miíase oral tem características clínicas típicas e diagnóstico simples, o qual é geralmente feito por meio da observação da movimentação das larvas (CHICARELLI et al., 2002). Dores, febre, odor acentuado e desconforto local são os principais sintomas relatados pelos portadores (KHAYAT, 2002; ABDO et al., 2006; HAKIMI; YAZID, 2002; DROMA et al., 2007; ROSSI-SCHNEIDER et al., 2007; STEPHAN; FUENTEFRIA, 1999; BARBOSA et al., 2008; CAVALCANTI et al., 2008).
Embora a miíase oral seja uma condição rara, o diagnóstico precoce e atendimento imediato faz-se necessário para reduzir os danos tissulares e propiciar uma rápida recuperação do paciente (FIGUEIREDO et al., 2000). Em casos mais graves, pode ocorrer envolvimento ósseo (KONSTANTINIDIS; ZAMANIS, 1987). Esta patologia pode se manifestar em feridas resultantes de exodontias e também em bolsas periodontais (LEAL et al., 2001), podendo ainda estar associada com outras patologias como a Noma [cancrum oris] (AGUIAR et al., 2003).

Em relação à localização na cavidade oral, a maior frequência é na região anterior da maxila e no palato, mas pode acometer também a região posterior (HAKIMI; YAZID, 2002; KHAYAT, 2002; NG et al., 2003; ROSSI-SCHNEIDER et al., 2007; DROMA et al., 2007; SHARMA et al., 2008; BARBOSA et al., 2008) e a língua (OLIVEIRA; MACHADO; OLIVEIRA, 2008; CAVALCANTI et al., 2008). Uma das hipóteses que podem explicar a maior frequência de relatos envolvendo a região ântero-superior é a presença do hábito de respiração bucal, uma vez que nos portadores desta condição essa região se torna mais facilmente exposta à deposição dos ovos pelas moscas.

A remoção mecânica das larvas é o tratamento mais comumente preconizado (ABDO et al., 2006; BHATT; JAYAKRISHNAN, 2000; GURSEL et al., 2002; HAKIMI; YAZID, 2002; DROMA et al., 2007; SHARMA et al., 2008; BARBOSA et al., 2008; AGUIAR et al., 2003). No entanto, outras modalidades terapêuticas são descritas e baseiam-se no emprego de substâncias químicas. Dentre essas, a mais comumente utilizada é a colocação de éter sobre a área lesionada (BHATT; JAYAKRISHNAN, 2000; CAVALCANTI et al., 2008). A aplicação dessa substância ocasiona a asfixia da larva, possibilitando mais facilmente a sua remoção (FIGUEIREDO et al., 2000).

Frente a casos de maior gravidade, além da remoção mecânica, o cirurgião-dentista deve fazer uso de antibióticos de largo espectro, sendo a ivermectina - droga antiparasitária de amplo espectro - o mais comumente utilizado (ABDO et al., 2006; SHINOHARA et al., 2004; VICTORIA et al., 1999; GEALH et al., 2009), devido a sua eficiência e no tratamento da miíase (SHARMA et al., 2008), além de apresentar uma absorção rápida, com elevada concentração sanguínea em relativamente pouco tempo. Atua, interfere na transmissão de impulsos nervosos, junto às terminações nervosas, por estímulo à liberação do ácido gama amino butírico [GABA] (SHINOHARA et al., 2004).

\section{Conclusão}

Quando diagnosticada precoce e corretamente, a miíase oral pode ser facilmente tratada pelo cirurgião-dentista, por meio da remoção mecânica das larvas com o auxílio ou não de substâncias químicas de uso local, apresentando um prognóstico favorável. Todavia, qualquer que seja o meio empregado, é indispensável que o paciente seja orientado quanto à adoção de medidas adequadas de higiene pessoal.

\section{Referências}

ABDO, E. N. Oral Myiasis: a Case Report. Med. Oral Patol. Oral Cir. Bucal., Valencia, v. 11, no. 2, p. E130-E131, Mar. 2006.

AGUIAR, A. M. M.; ENWONWU, C. O.; PIRES, F. R. Noma (Cancrum oris) Associated to Oral Myiasis in an Adult. Oral Dis., Copenhagen, v. 9, no. 3, p. 158-159, May 2003.

BARBOSA, T. de S. et al. Oral Infection by Diptera Larvae in Children: a Case Report. Int. J. Dermatol., Philadelphia, v. 47, no. 7, p. 696699 , July 2008. 
BHATT, A. P.; JAYAKRISHNAN, A. Oral Myiasis: a Case Report. Int. J. Paediatr. Dent., Oxford, v. 10, n. 1, p. 67-70, Mar. 2000.

BOZZO, L. et al. Oral Myiasis Caused by Sarcophagidae in an Extraction Wound. Oral Surg. Oral Med. Oral Pathol., St. Louis, v. 74 n. 6, p. 733-735, Dec. 1992.

CAVALCANTI, A. L. et al. Oral Myiasis. Stom. Glas S, Belgrado, v. 55, n. 4, p. 254-258, Oct./Dec. 2008.

CENCIL, J. et al. Miíase Bucal: Revisão de Literatura. Publ. UEPG: Ci. Biol. Saúde, Ponta Grossa, v. 12, n. 2, p. 39-43, jun. 2006.

CHICARELLI, M. et al. Miíase Humana Bucal por Cochliomyia Hominivorax (Coquerel, 1858) em Nova Esperança, Estado do Paraná, Brasil. RFO UFP: Rev. Fac. Odontol. Univ. Passo Fundo, Passo Fundo, v. 7, n. 2, p. 39-41, jul./dez. 2002.

DROMA, E. B. et al. Oral Myiasis: a Case Report and Literature Review. Oral Surg. Oral Med. Oral Pathol. Oral Radiol. Endod., St. Louis, v. 103, no. 1, p. 92-96, Jan. 2007.

FERRO, A. P. N. et al. Human Been Myiasis in the Uberlandia Region, State of Minas Gerais, Brazil. Biosci. J., Uberlândia, v. 19, n. 1 , p. 77-81, jan./abr. 2003.

FIGUEIREDO, M. C. et al. Miíase Oral: Relato de um Caso Clínico. J. Bras. Odontoped. Odonto Bebê, Curitiba, v. 3, n.13, p. 243-246, maio/jun. 2000.

FREITAS, R. R. et al. Míase da Cavidade Bucal: Relato de um Caso. Rev. Assoc. Paul. Cir. Dent., São Paulo, v. 43, n. 6, p. 291-294, nov./ dez. 1989

GEALH, W. C. et al. Treatment of Oral Myiasis Caused by Cochliomyia Hominivorax: Two Cases Treated with Ivermectin. Br. J. Oral Maxillofac. Surg., Edinburgh, v. 47, no. 1, p. 23-26, Jan. 2009.

GURSEL, M. et al. A Rare Case of Gingival Myiasis Caused by Diptera (Calliphoridae). J. Clin. Periodontol., Copenhagen, v. 29, no. 8, p. 777-780, Aug. 2002.

GUTIERREZY. Diagnostic Pathology of Parasitic Infection with Clinical Correlations. Philadelphia: Lea \& Febiger, p. 489-496, 1990.

HAKIMI, R.; YAZID, I. Oral Mucosa Myiasis Caused by Oestrus Ovis. Arch. Iranian Med., Tehran, v. 5, no. 3, p. 194-196, July 2002.

HALL, M. J. R.; WALL, R. Myiasis of Humans and Domestic Animals. Adv. Parasitol., London, v. 35, p. 257-334, 1995.

JOO, C. Y.; KIM, J. B. Nosocomial Submandibular Infections with Dipterous Fly Larvae. Korean J. Parasitol., Seoul, v. 39, no. 3, p. 255260, Sept. 2001.

KHAYAT, R. M. A Case Report on Oral Myiasis in Saudi Arabia. Saudi Dental. J., Riyadh, v. 14, n. 3, p. 140-142, Sept./Dec. 2002.

KONSTANTINIDIS, A. B.; ZAMANIS, D. Gingival Myiasis. J. Oral Med., New York, v. 42, n. 4, p. 243-245, Oct./Dec. 1987.

LEAL, J. L. F. et al. Miíase Orofacial: Relato de um Caso. Rev. Fac. Odontol. UFBA, Salvador, v. 22, n. 2, p. 95-99, jul./dez. 2001.

LIM, S. T. Oral Myiasis: a Review. Singapore Dent. J., Singapore, v. 13, n. 2, p. 33-34, May 1974.
MEINKING, T. L.; BURKHART, C. N.; BURKHART, C. G. Changing Paradigms in Parasitic Infections: Common Dermatological Helminthic Infections and Cutaneous Myiasis. Clin. Dermatol., Philadelphia, v. 21, n. 5, p. 407-416, Sept./Oct. 2003.

MELO, R. E. V. A.; VÍTOR, C. M. A.; PINTO, S. F. Miíases Humanas. An. Fac. Odontol. Univ. Fed. Pernambuco, Recife, v. 10, n. 1, p. 7379, jan./jun. 2000.

MILLIKAN, L. E. Myiasis. Clin. Dermatol., Philadelphia, v. 17, no 2 , p. 191-195, Mar./Apr. 1999.

NG, K. H. L. et al. A Case of Oral Myiasis Due to Chrysomya Bezziana. Hong Kong Med. J., Hong Kong, v. 9, n. 6, p. 454-456, Dec. 2003.

OLIVEIRA, J. A. G. P.; MACHADO, M. I.; OLIVEIRA, M. P. Miíase de Língua: Relato de um Caso de Infestação Hospitalar. Rev. Cir. Traumatol. Buco-Maxilo-Fac., Recife, v. 8, n. 4, p. 47-50, out./dez. 2008.

PONTES, F. S. C. et al. Miíase Gengival: Relato de um Caso Clínico. J. Bras. Clin. Odontol. Integr., Curitiba, v. 6, n. 32, p. 151-153, mar./ abr. 2002.

RAWLINS, S. C.; BARNETT, D. B. Internal Human Myiasis. West Indian Med. J., Mona, Jamaica, v. 32, n. 3, p. 184-186, Sept. 1983.

ROSSI-SCHNEIDER, T. et al. Oral Myiasis: a Case Report. J. Oral Science, Tokyo, v. 49, n. 1, p. 85-88, Mar. 2007.

SCOTT, H. G. Human Myiasis in North America. Florida Entomol., Gainesville, v. 47, p. 255, 1964.

SHARMA, J.; MAMATHA, G. P.; ACHARYA, R. Primary Oral Myiasis: a Case Report. Med. Oral Patol. Oral Cir. Bucal, Valencia, v. 13, n. 11, p. E714-E716, nov. 2008.

SHINOHARA, E. H. et al. Oral Myiasis Treated with Ivermectin: Case Report. Braz. Dent. J., Ribeirão Preto, v. 15, n. 1, p. 79-81, 2004.

STEPHAN, A.; FUENTEFRIA, N. B. Miíase Oral: Parasita Versus Hospedeiro. Rev. Assoc. Paul. Cir. Dent., São Paulo, v. 53, n. 1, p. 47-49, jan./fev. 1999 .

VICTORIA, J.; TRUJILLO, R.; BARRETO, M. Myiasis: a Successful Treatment with Topical Ivermectin. Int. J. Dermatol,, Philadelphia, v. 38, no. 2, p. 142-144, Feb. 1999.

YAZAR, S. et al. Nosocomial Oral Myiasis by Sarcophaga sp. in Turkey. Yonsei Med. J., Seoul, v. 46, n. 3, p. 431-434, June 2005. 